\title{
A Study of Blended Learning Design to Promote Deep Learning
}

\author{
Yanmei Zhou 1, a, Zhi Zhang 2, b \\ ${ }^{1}$ School of Physics and Electronics, HUNAN University, Changsha 410082, China; \\ ${ }^{2}$ School of Physics and Electronics, HUNAN University, Changsha 410082, China; \\ a shuzimeijie@163.com, b632027503@qq.com
}

\begin{abstract}
Keywords: deep learning, blended leaning, Instructional design.
\end{abstract}
\begin{abstract}
As the main research content and important teaching ideas of learning science, deep learning has become the focus of researchers. Blended learning is one of the important directions of teaching reform and is closely linked with deep learning. Based on the analysis of the connotation features of deep learning and blended learning, the article explains the connotation of blended learning that promotes deep learning, and focuses on the promotion of deep learning from the aspects of front-end analysis, organization and activity design of teaching resources, and teaching evaluation. It provides theoretical and practical guidance for deep learning and blended leaning.
\end{abstract}

\section{Introduction}

With the development of the Internet and information technology, new changes have taken place in teaching concepts and learning methods, and the goal of pursuing teaching effectiveness has remained unchanged. However, in traditional classroom teaching and simple online learning, there is a simple mechanical rote learning surface learning that seriously hampers students' deep understanding of knowledge and the development of higher-order thinking skills such as critical thinking, problem solving, and innovative thinking. Deep learning, as an important trend in the development of education, coincides with the training of talent in the 21 st century. Therefore, it is of great significance to promote deep learning of learners. At present, the teaching methods tend to be combined with online and offline. Especially with the development of micro lessons, MOOCs (massive open online courses), SPOC (Small Private Online Courses), and flipped classrooms, blended learning has gradually become one of the most important directions in college teaching reform. Blended learning complements the advantages of traditional classroom teaching and online learning in order to achieve the optimal learning effect [1], which is in line with the purport of deep learning. However, how to design a blended learning to promote students' deep learning has become a problem that needs to be solved urgently. Therefore, on the basis of analyzing the connotation features of deep learning and mixed teaching, the article explains the connotation of the blended learning that promotes deep learning, and focuses on exploring the blended learning design that promotes deep learning, with a view to providing a reference for promoting blended learning design for deep learning.

\section{Deep Learning and Blended Learning}

\subsection{Deep Learning}

Deep learning originated in the study of reading strategies of college students by Swedish scholars Ference Marton and Roger Saljo in the mid-1950s. The study found that learners have different levels of processing of learning materials when they receive learning materials, namely deep learning and surface learning. Surface learning tends to memorize and understand mechanically, while deep learning tends to understand the use of migration on the basis of high level cognitive level in Bloom's educational goals, with emphasis on analysis, synthesis, and evaluation [2] .The earliest introduction of deep learning concepts into China was Professor Li Jiahou, who proposed that deep learning is based on the understanding of learning, learners critically learn new content, and integrate new knowledge into the original cognitive structure. The old and new knowledge are linked and can be 
used in new contexts to make decisions and solve problems [3]. The research on deep learning at home and abroad is mainly concentrated in the computer field and education field. The common consensus in the field of education is that it is a new learning concept in the information age and a learning style relative to surface learning. The Deep Learning Study Project (SDL) conducted by the William and Flora Hewlett Foundation broke the cognitive limit and defined deep learning as the ability that students must possess in the 21 st century work and citizen life. A deep learning ability framework composed of cognitive, interpersonal, and personal domains provides guidance for further advancement and innovation of deep learning [4]. In order to understand deep learning, besides the cultivation of core subject knowledge and thinking at the cognitive level, the development of individual ability should also be emphasized so that students can learn to learn.

\subsection{Blended Learning}

Blending Learning, is a new teaching method formed after a profound reflection on traditional face-to-face teaching and distance online learning, which first emerged in the field of corporate training. He Kekang believes that: Blending Learning is to combine the advantages of traditional learning methods with the advantages of e-Learning. It not only plays a leading role in guiding, inspiring, and monitoring the teaching process of teachers, but also fully embodies the initiative, enthusiasm, and creativity of students as the subject of teaching [1]. Curtis Bunker believes that blended learning is the combination of face-to-face teaching and computer-assisted online learning [5], this concept is generally accepted. Blended learning forms are diversified and need to be mixed according to different learning objects, learning content, learning needs, and learning situations, and have achieved optimal teaching results. At present, the research of mixed teaching based on digital resources (micro-curriculum, MOOC, SPOC) and new teaching platform emerges in an endless stream. The flipped classroom is regarded as the representative of blended learning.

\subsection{Promoting Deep Learning Blended Teaching Connotation}

Blended learning aims to achieve the optimization of teaching effects through the complementary advantages of online and offline. To a certain extent, it is the pursuit of students' deep learning. Deep learning is the potential requirement for mixed teaching. At the same time, the blended learning has a unique combination of learning resources, learning environments, learning styles, and learning assessments, and it has unique advantages and support for promoting students' in-depth learning. Therefore, it is necessary and feasible to promote students' deep learning by blended learning. Through the guidance of deep learning theory and combining the characteristics of blended learning, the objectives of teaching across the cognitive level will be further clearly blended, and the deep learning ability of the learners' motor skills, interpersonal, and individual levels will be further developed. The blended learning that promotes deep learning is to promote students to achieve deep learning and cultivate students' in-depth learning ability through a combination of teaching methods based on deep learning goals. The deep learning theory is merged to guide the blended learning design, blended learning forms are diversified and taught for deep learning.

\section{Promote Mixed Learning Design for Deep Learning}

The characteristics of deep learning mainly focus on critical understanding, emphasizing information integration, promoting knowledge construction, intentional migration, problem solving, and proactive life [6]. The deep competence framework proposed by the SDL project provides a clear teaching goal and evaluation basis for the implementation of mixed teaching. However, the question of how tactical instruction promotes deep learning of mixed instructional design is a question worth exploring. Therefore, this study combines the deep learning line [7] proposed by Eric Jensen and LeAnn Nickelsen and designs blended learning that promotes deep learning mainly from the aspects of front-end analysis, teaching resource organization and activity design, and teaching evaluation. The following will elaborate on the blended learning design that promotes deep learning.

\subsection{Deep Front-End Analysis, Comprehensive Reconstruction of the Curriculum}

In the instructional design, the front-end analysis serves as the starting point and foundation of the instructional design. The front-end analysis needs to focus on the characteristics of the learner, the content of the teaching, and the teaching objectives. In the blended learning design, we must take a 
deeper understanding of the learner's status of deep learning. At the same time, combined with the learner's existing knowledge level, learning style and other characteristics, grasp the design of teaching resources and content. According to the revised Bloom's cognitive target classification and knowledge classification theory, the teaching content is refined, and under the guidance of deep learning objectives, the depth of knowledge is defined, and targeted learning resources for deep learning are targeted. From the top-level design, the overall reconfiguration of the curriculum includes the contents and forms of the curriculum, and the corresponding curriculum materials and teaching methods. In addition, according to actual needs, it is necessary to reconstruct the blended learning environment and create a campus deep learning culture. The overall goal is to achieve students' deep learning, reconstruct curriculum, and conduct overall design.

\subsection{Organize Personality Resources and Initiate Autonomy-Collaboration-Exploration}

Teaching resources and teaching activities are the main carriers for achieving student learning, and they are also an important part of students' deep learning. According to the front-end analysis, learners have different characteristics and the need for learning, so the organization of personalized and diverse teaching resources is indispensable, the content and form need to pay special attention to the convergence and learning aids before and after, different stages play different roles, such as preschool inspectors, students in class, after school Consolidate the expanded functionality. The design of teaching activities should also be based on the mechanism of learning, match learning unit activities, and use deep learning strategies such as problem-based learning, project-based learning, inquirybased learning, and collaborative learning. Process evaluation, etc., guide students to form a deeplearning model of self-coordination-inquiry integration. In specific teaching activities, students are encouraged to construct knowledge, and students are encouraged to understand and master core subject knowledge and develop higher-order thinking. In the implementation of teaching, suitable cognitive tools and deep learning strategies can assist students in deep learning, such as writing reflective notes, introducing concept maps, mind maps, and notes in teaching. Tools, etc. Situation, experience, interaction, and reflection are the four elements of the deep learning process. During the implementation of teaching activities, situations should be reasonably created, students' experiences should be emphasized, and multiple interactions and student reflection should be strengthened. The advantages of the online platform should be fully utilized. At the same time, encourage group collaboration, establish a learning community, and provide necessary learning support in the learning process, to achieve personalized deep learning. In general, for the design of resource organization and teaching activities in blended learning, it is necessary to consider both online and offline. In the specific implementation, according to the depth learning route [7], incorporate online and offline organic integration to support students in deep learning.

\subsection{Continuously Multiple Evaluations, Guaranteed Achievement}

Teaching evaluation is closely linked with teaching objectives. In the blended learning design that promotes deep learning, the result or standard of teaching evaluation is the achievement of students' deep learning. In addition to diagnostic functions, teaching evaluation also has development functions. Therefore, the deep learning evaluation is accompanied by the entire blended learning process, which promotes learning. The combination of process evaluation, summative evaluation, and self-evaluation, mutual evaluation, and teacher evaluation. In the evaluation of various levels of deep learning, professional scales and measurement tools are selected to perform online and offline performance to ensure that learners achieve deep learning. The online learning platform provides the basis for the final learning evaluation and can also serve as data support for the teaching diagnosis and dynamic tracking research. When a large amount of data is accumulated, data analysis and mining can be conducted to find teaching problems and guide instructional design. Continuous and multiple evaluation can effectively stimulate learning and ensure the achievement of teaching effects

\section{Conclusion}

Based on the analysis of the connotation of deep learning and blended learning, and the relationship between deep learning and blended learning, this study explores the design of blended learning that promotes deep learning, with a view to providing references for promoting blended 
learning in deep learning. In the next step, we will combine in-depth empirical research to further build and validate a deep learning model in a blended learning environment.

\section{References}

[1]. He Keqiang. Looking at the new development of educational technology theory from Blending Learning (I) [J]. e-Education Research, 2004(03):1-6.

[2]. Marton F,Saljo R. On qualitative differences in learning: I-Outcome and process[J]. British Journal of Educational Psychology, 1976, (46):4-11.

[3]. He Ling, Li Qiang. Promote students' deep learning [J]. Modern Teaching,2005, (5):29-30.

[4]. Bu Caili, Feng Xiaoxiao, Zhang Baohui. Concept, Strategy, Effect and Inspiration of Deep Learning--Interpretation and Analysis of US Deep Learning Project (SDL) [J]. Journal of Distance Education, 2016,34(05):75-82.

[5]. Zhan Zehui, Li Xiaohua. Mixed Learning: Definitions, Strategies, Status Quo, and Trends of Development - A Conversation with Professor Curtis Bunker of Indiana University, USA [J]. China Educational Technology,2009,(12):1.

[6]. Zhang Hao, Wu Xiujuan. Connotation of deep learning and the theoretical basis of cognitive theory [J]. China Educational Technology,2012(10):7-11+21.

[7]. Eric Jensen, LeAnn Nickelsen. 7 powerful strategies for deep learning [M]. Shanghai: East China Normal University Press, 2010. 